\title{
COMMENTARY
}

\section{Traumatic cardiac arrests - the action or the provider, what makes the difference?}

\author{
Kelly R Klein* \\ See related viewpoint by Sherren et al., http://ccforum.com/content/17/2/308
}

\begin{abstract}
Traumatic cardiac arrest resuscitation is considered a heroic and futile endeavor. However, newer articles have more promising statistics and divide between prehospital ground and helicopter transport. Here we discuss why there might be a difference in the survivability of this subset of trauma patients.
\end{abstract}

According to popular studies, the attempted resuscitation of patients in traumatic cardiac arrest in the prehospital care environment should not be pursued due to undo risk to the provider and has been shown to be ineffective. However, after review of current literature, Sherren and colleagues developed and published in a recent edition of Critical Care a detailed treatment algorithm for their helicopter emergency medical service (HEMS) that encourages aggressive resuscitation for patients in traumatic cardiac arrest (TCA) [1]. What is known in a review of the quoted literature is that in the prehospital care environment, the survival rate of TCA patients is 0 to $3.7 \%$, but in newer published studies survivability has risen to $7.5 \%[2,3]$.

What we already know is that prehospital medical cardiac arrest (MCA) survival is approximately 9.8\%, with in-hospital cardiac arrest survival at $24.2 \%$ [4]. In MCA, chest compressions, defibrillation, medication, and oxygenation are the mainstay treatment tools. Where the TCA patient differs is that many do not have extensive co-morbidities or coronary artery disease; their arrest is primarily due to one of or a combination of factors: hypovolemia, obstruction of blood flow and hypoxia [5]. In the patient with thoracic trauma, these causes are addressed by well described techniques of

${ }^{*}$ Correspondence: Kelly.Klein@utsouthwestern.edu

UT Southwestern at Dallas, Division of Emergency Medicine, Section of EMS/

Disaster Medicine/Homeland Security, 5323 Harry Hines Blvd, MC 8890, Dallas, TX 75390-8890, USA thoracostomies, endotracheal intubation and blood products. This is followed by a clamshell thoracotomy, allowing for a better chance for hemorrhage control and treatment of hemopericardium if found. Sherren and colleagues discuss a very well outlined pathophysiology and rationale for TCA survivorship in their article.

Although MCA and TCA are not the same diseases, their survival statistics are interesting and possibly some inference can be made when MCA data are more closely observed. It appears that the differences between MCA survival in the prehospital versus a hospital setting, where admitted patients are older with increased morbidities, might be due to the personnel performing the resuscitation. Staffing models for emergency medical services (EMS), especially for HEMS, differ between the US system and the European model. In the US, prehospital EMS systems (including most HEMS) are staffed by allied health professionals and not by physicians. In the European model (including Australia) HEMS are staffed by well trained physician/paramedic/nurse teams and this is possibly where the difference occurs for survival for TCA patients.

In multiple European studies it was noted that there was a decrease in mortality of trauma patients without a decrease in scene time when a physician was part of the flight crew [6-9]. In the US, less than 5\% of HEMS are staffed by physicians, and most of those involved in such teams are in their first few years out of medical school [10]. Thus, no US EMS systems have fully trained physicians as part of their standard ambulance crew. A reasonable question to consider is whether staffing is the reason why TCA statistics differ between the US and European models? Paramedic training teaches needle thoracostomy for suspected tension pneumothorax, which in many jurisdictions do not need online direct physician permission as it is deemed life saving and with limited risk [11]. However, it is well known that in patients with larger chest walls, almost $50 \%$ of the time the needle fails to reach the plural space to relieve the obstruction of blood flow caused by the tension pneumothorax. On the contrary, the placement of a tube or finger thoracostomy are very successful and fairly 
routine in EMS systems staffed with experienced physicians with excellent results [12].

The HEMS algorithm of Sherren and colleagues is specific for flight physicians. It cannot be fully applicable to HEMS not staffed by a well trained physician, as the proficiency of thoracotomy is an advanced skill that takes practice and years of anatomical training to be able to perform well. As the authors stated, more study is needed to see if it is the depth of training or the protocols that are the reason for the increases in survivorship from TCA. I do not believe that this algorithm will be promoted in HEMS systems staffed only by paramedics and nurses, as their level of training does not allow for advanced procedures such as these. Therefore, one should expect that prehospital attempts at the resuscitation of the TCA in those systems without physicians on board will remain futile.

\section{Abbreviations}

EMS, emergency medical services; HEMS, helicopter emergency medical service; MCA, medical cardiac arrest; TCA, traumatic cardiac arrest.

\section{Competing interests}

The author declares that they have no competing interests.

Published: 17 June 2013

\section{References}

1. Sherren PB, Reid C, Habig K, Burns BJ: Algorithm for the resuscitation of traumatic cardiac arrest patients in a physician-staffed helicopter emergency medical service. Crit Care 2013, 13:308.

2. Lockey D, Crewdson K, Daview G: Traumatic cardiac arrest: who are the survivors? Ann Emerg Med 206, 48:240-244.
3. Pickens JJ, Copass MK, Bulger EM: Trauma patients receiving CPR: predictors of survival. J Trauma 2005, 58:951-958.

4. Go AS, Mozaffarian D, Roger VL, Benjamin EJ, Berry JD, Borden WB, Bravata DM, Dai S, Ford ES, Fox CS, Franco S, Fullerton HJ, Gillespie C, Hailpern SM, Heit JA, Howard VJ, Huffman MD, Kissela BM, Kittner SJ, Lackland DT, Lichtman JH, Lisabeth LD, Magid D, Marcus GM, Marelli A, Matchar DB, McGuire DK, Mohler ER, Moy CS, Mussolino ME, et al.: Heart disease and stroke statistics - 2013 update: a report from the American Heart Association. Circulation 2013, 127:e6-e245.

5. Stockinger ZT, MCSwain NE: Additional evidence in support of withholding terminating cardiopulmonary resuscitation for trauma patients in the field. J Am Coll Surg 2004, 198:227-231.

6. Baxt WG, Moody P: The impact of a physician as part of the aeromedical prehospital team in patients with blunt trauma. JAMA 1987, 257:3246-3250.

7. Matsumoto H, Mashiko K, Hara Y, Sakamoto Y, Kutsukata N, Takei K, Tomita Y, Ueno Y, Yamamoto Y: Effectiveness of a "doctor-helicopter" system in Japan. Israel Med Assoc J 2006, 8:8-11.

8. Garner A, Rashford S, Lee A, Bartolacci R: Addition of physicians to paramedic helicopter services decreases blunt trauma mortality. Aust N Z J Surg 1999, 69:697-701.

9. Dissmann PD, Le Clerc S: The experience of Teesside helicopter emergency services: doctors do not prolong prehospital on-scene times. Emerg Med J 2007, 24:59-62

10. Bjoernsen LP: "Doctors in the Air"; do we need them, and if so, how should we train them? Internet J Aeromed Transportation 2010, 2. DOI: 10.5580/19d4

11. Martin M, Satterly S, Inaba K, Blair K: Does needle thoracostomy provide adequate and effective decompression of tension pneumothorax? J Trauma Acute Care Surg 2012,73:1410-1415.

12. Massarutti D, Trillò G, Berlot G, Tomasini A, Bacer B, D'Orlando L, Viviani M, Rinaldi A, Babuin A, Burato L, Carchietti E: Simple thoracostomy in prehospital trauma managment is safe and effective: a 2-year experience by helicopter emergency medical crew. Eur J Emerg Med 2006, 13:276-280.

doi:10.1186/cc12720

Cite this article as: Klein KR: Traumatic cardiac arrests - the action or the provider, what makes the difference? Critical Care 2013, 17:156. 